УДК 550.837.6

\title{
ПРИМЕНЕНИЕ ПСЕВДОВОЛНОВЫХ ТРАНСФОРМАНТ В ИМПУЛЬСНОЙ ЭЛЕКТРОРАЗВЕДКЕ ДЛЯ ВЫДЕЛЕНИЯ НАКЛОННЫХ ГЕОЛОГИЧЕСКИХ ГРАНИЦ
}

\author{
Грецков Глеб Андреевич',
} Greckov.Gleb@gmail.com

Эпов Михаил Иванович', EpovMl@ipgg.sbras.ru

\author{
Шеин Александр Николаевич', \\ SheinAN@ipgg.sbras.ru \\ Институт нефтегазовой геологии и геофизики им. А.А. Трофимука Сибирского отделения РАН, \\ Россия, 630090, г. Новосибирск, пр Ак. Коптюга, 3.
}

Актуальность. Метод нестационарных электромагнитных зондирований или зондирований становлением поля является востребованным инструментом при поиске и разведке месторождений различного типа полезных ископаемых. Современные задачи электроразведки требуют использования установок с большим числом источников и приёмников. Для подобного типа установок задача интерпретации полученных данных является весьма ресурсоемкой. Отсюда вытекает острая необходимость развития методов обработки и интерпретации данных, полученных в результате импульсных электромагнитных зондирований. Одним из возможных путей такого развития могут послужить трансформации данных зондирований становлением в пространство решений волнового уравнения.

Цель: применить метод волновой трансформации к данным зондирований становлением, зарегистрированным над проводящими средами с наклонной границей в многоразносных установках, для получения новых качественных оценок исследуемого геоэлектрического разреза.

Методика исследования базируется на новых численных алгоритмах для устойчивого преобразования данных зондирований становлением в волновую область. Исходные данные для отладки процедур трансформации (расчётные кривые становления поля) были получены с использованием программного комплекса МODEM3D, который предназначен для прямого моделирования переходных процессов диффузии электромагнитных полей.

Результаты. Были получены псевдоволновые трансформанты сигналов нестационарных электромагнитных зондирований в средах с наклонной границей, и построены их годографы. Полученные наборы трасформаций позволяют выделить линию выхода наклонного контакта на дневную поверхность. Установлено, что перекрывающие наносы ослабляют максимумы псевдоволновых трансформаций и снижают возможность точной локализации линии выхода наклонной границы. Рассмотрено поведение тороидальных вихревых токов в зависимости от времени для модели геологической среды с наклонной границей.

\section{Ключевые слова:}

Нестационарные электромагнитные зондирования, псевдоволновые трансформанты,

Q-преобразование, трёхмерная прямая задача, среда с наклонной границей, годограф.

\section{Введение}

В разведочной геофизике широко применяется метод нестационарных электромагнитных зондирований или зондирований становлением поля (3C), основанный на изучении временных развёрток откликов от импульсного токового возбуждения проводящих геологических сред. Он является востребованным инструментом как при геологоструктурных исследованиях и геологическом картировании, так и при поисках и разведке месторождений различного типа полезных ископаемых. При этом для достоверного построения пространственной структуры подземного полупространства необходимо использовать весьма ресурсоёмкое численное моделирование решений как прямых, так и обратных трёхмерных задач электродинамики. Для восстановления структуры среды применяется широкий спектр процедур инверсии измеренных на дневной поверхности компонент магнитной индукции (или ЭДС, наведённой в измерительных контурах). В этой связи оказываются чрезвычайно полезными различные трансформации из- меренных сигналов в кажущееся удельные электрические сопротивление $\rho_{\tau}$ [1] или зависимости кажущейся продольной проводимости $S_{\tau}$ от кажущейся глубины $H_{\tau}$ [2]. В связи с многократно возросшей производительностью процессоров стало возможным создание процедур преобразования сигнала, описывающегося уравнением диффузии, в трансформанты, удовлетворяющие волновому уравнению (псевдоволновые трансформанты). Применение этого преобразования позволяет не только составить достоверное представление о строении исследуемой геологической среды, но и дополнять традиционные методы интерпретации [3].

\section{Численное решение прямых задач 3С}

Для прямого моделирования переходных процессов диффузии электромагнитных полей от замкнутых токовых контуров в проводящих средах использовалась программа MODEM3D [4]. Соответствующая прямая задача в квазистационарном приближении решается векторным методом конечных элементов на неструктурированной трёх- 
мерной тетраэдральной сетке. Интегрирование по времени выполняется с применением неявной схемы Кранка-Николсона второго порядка точности.

Повышение производительности вычислений базируется на распараллеленных алгоритмах, использующих многоядерные процессоры. Для массовых расчётов программа адаптирована к работе в системе распределённых вычислений (GRID-система) и облачных средах, организованных в ИНГГ СО РАН [5]. Тестирование на горизонтально-слоистых средах с простыми по форме неоднородностями показало, что для широкого класса моделей расчёты выполняются с высокой точностью [6].

\section{Численное моделирование псевдоволновых трансформант}

Для преобразования данных 3 С воспользуемся отображением пространства решений уравнения диффузии $V$ в пространство решений волнового уравнения $U$. Это можно выполнить, используя преобразование Лапласа [7, 8]:

$$
B(t)=\frac{1}{2 \sqrt{\pi t}} \int_{0}^{\infty} q e^{\frac{-q^{2}}{4 t}} U(q) d q .
$$

В этом интегральном уравнении $B$ - составляющая магнитной индукции переходного процесса, $U$ $(q)$ - её псевдоволновая трансформанта, $q^{2}$ - аналог времени (приведённое время) в пространстве $U$. При преобразовании (1) остаётся неопределенность в выборе переменной приведённого времени. Её доопределение может быть выполнено исходя из физических или каких-либо иных соображений. Для нахождения псевдоволновой трансформанты необходимо решить обратную задачу с интегральным оператором. Известно, что её решение с экспоненциально затухающим при возрастании аргумента $q$ ядром является неустойчивым и необходимо использовать различные методы регуляризации.

\section{Обзор методов получения псевдоволновых трансформант}

В работе [9] для данных электромагнитных зондирований с контролируемым источником (CSEMControlled Source ElectroMagnetic) применено сингулярное разложение, а полученное «недиффузионное» решение использовано для томографической инверсии. Хотя такой подход приводит к некоторым полезным результатам, трансформанты оказались сильно осциллирующими и непригодными для методов обработки сейсмических сигналов. В работе [10] показан способ стабилизации преобразования путём минимизации его $L_{1}$-нормы методами линейного программирования. Другие попытки стабилизации с отображением пространства решений диффузионного уравнения в пространство решений волнового были предприняты в работах $[11,12]$. В них в основном использовались методы разложения поля на базисные функции, для которых преобразование (1) может быть выполнено в аналитическом виде. Все они базируются только на критерии вычислительной стабильности и не учитывают желаемые физические свойства результирующих псевдоволновых трансформант. Проблемы регуляризации решения интегрального уравнения (1) подробно описаны в диссертационной работе [13]. В ней обосновны и применены для данных CSEM SVD-регуляризация и регуляризация Тихонова с различными параметрами. В работе [14] был проведён теоретический анализ и представлены численные результаты преобразования данных $3 \mathrm{C}$ в псевдоволновые трансформанты. На их временных развёртках выделены эффекты отражения и рефракции. В работе [15] были проанализированы причины пространственной дисперсии псевдоволновых трансформант и представлена методика их уточнения с использованием деконволюции. По «исправленным» таким образом значениям точнее определяются границы погруженного проводящего объекта. Li X. Xue G. и Yin C описали метод увеличения пространственного разрешения для сигналов в установке с одним генераторным и несколькими приёмными контурами, основанный на принципе суперпозиции [16].

Для более точного вычисления псевдоволновых трансформант в работе [17] описан алгоритм субрегуляризации. Был рассмотрен альтернативный подход, основанный на так называемом обратном Q-преобразовании. В результате получаются трансформанты, удовлетворяющие стандартной системе волновых уравнений. Последние могут быть использованы в методах сейсмической миграции с более высоким пространственным разрешением целевых объектов для последующей прямой геологической интерпретации или уточнения начальной модели в инверсии.

В статьях $[18,19]$ рассмотрены примеры псевдоволновых трансформант нестационарной ЭДС в типичных горизонтально-слоистых моделях. Однако такой модельной базы недостаточно при изучении пространственно неоднородных геологических сред. Особенно это относится к средам с наклонными границами. В этом случае распределение и временная эволюция вихревых токов осложняется появлением поверхностных зарядов на этих границах и изменением степени затухания вторичных электромагнитных откликов во времени и пространстве. В результате инверсии таких данных в результирующей среде могут появяться ложные объекты или её восстановленная структура будет далека от истинной [20].

\section{Псевдоволновые трансформанты в средах} с наклонной границей

Рассмотрим контрастные геоэлектрические модели с наклонной границей, выходящей на плоскую дневную поверхность (верхнее полупространство предполагается непроводящим и немагнитным). Плоская контактная поверхность разделяет 

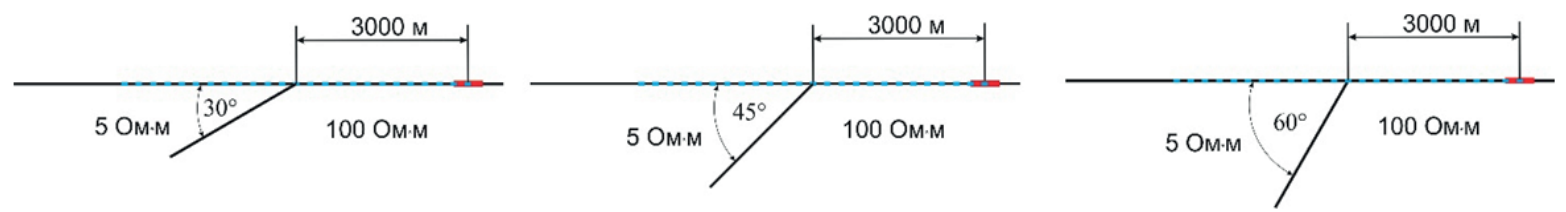

Рис. 1. Модели среды с наклонной границей

Fig. 1. Models of medium with inclined boundary

два проводящих немагнитных полубесконечных сектора с удельными электрическими сопротивлениями (УЭС) 5 и 100 Ом·м. Углы наклона границы составляют $30^{\circ}, 45^{\circ}$ и $60^{\circ}$ (рис. 1 ).

Выберем поверхностную установку, содержащую один источник и набор приёмников, расположенных на линейном профиле, перпендикулярном наклонной границе. Источник представляет собой квадратный токовый контур со стороной 500 м и расположен на поверхности среды с высоким УЭС $(100$ Ом·м). Его центр находится на удалении 3000 м от выхода наклонного контакта на поверхность. Приёмники - квадратные петли со стороной 100 м, расположены по профилю с шагом между центрами 50 м. Центр наиболее удалённого приёмника находится на расстоянии 6000 м на дневной поверхности проводящего (5 Ом·м) сектора.

Как известно [1], после мгновенного выключения постоянного тока силой $1 \mathrm{~A}$, текущего в генераторной петле, под дневной поверхностью возникает аналогичный по форме тороидальный токовый вихрь. С течением времени он распространяется вглубь и вширь проводящего полупространства, изменяя свою конфигурацию и амплитуду в зависимости от пространственного распределения
УЭС в среде. При этом интенсивность наведённых вихревых токов снижается за счёт перехода части электромагнитной энергии в джоулево тепло.

Введём декартову систему координат с началом в точке пересечения дневной поверхности, наклонного контакта и линейного профиля. Ось X совпадает с линейным профилем, а ось $\mathrm{Z}$ направлена вертикально вниз. На рис. 2, 3 показаны в вертикальном сечении (X0Z) распределения амплитуд электрического поля в различные моменты времени после выключения тока в источнике (показан тонким прямоугольником), вычисленные в программе MODEM3D.

На рис. 2 видно, что до момента времени $t=4,5$ мс электрический вихрь в виде тороида с квазикруговым сечением распространяется так же, как в однородном полупросранстве с УЭС 100 Ом·м (первая фаза распространения). Как неоднократно указывалось в литературе, линии перемещения центров электрического вихря наклонены под углом примерно \pm 26 градусов к дневной поверхности. После вышеуказанного момента времени появляется влияние наклонной границы (вторая фаза распространения). Оно выражается в существенном уменьшении глубины левого центра

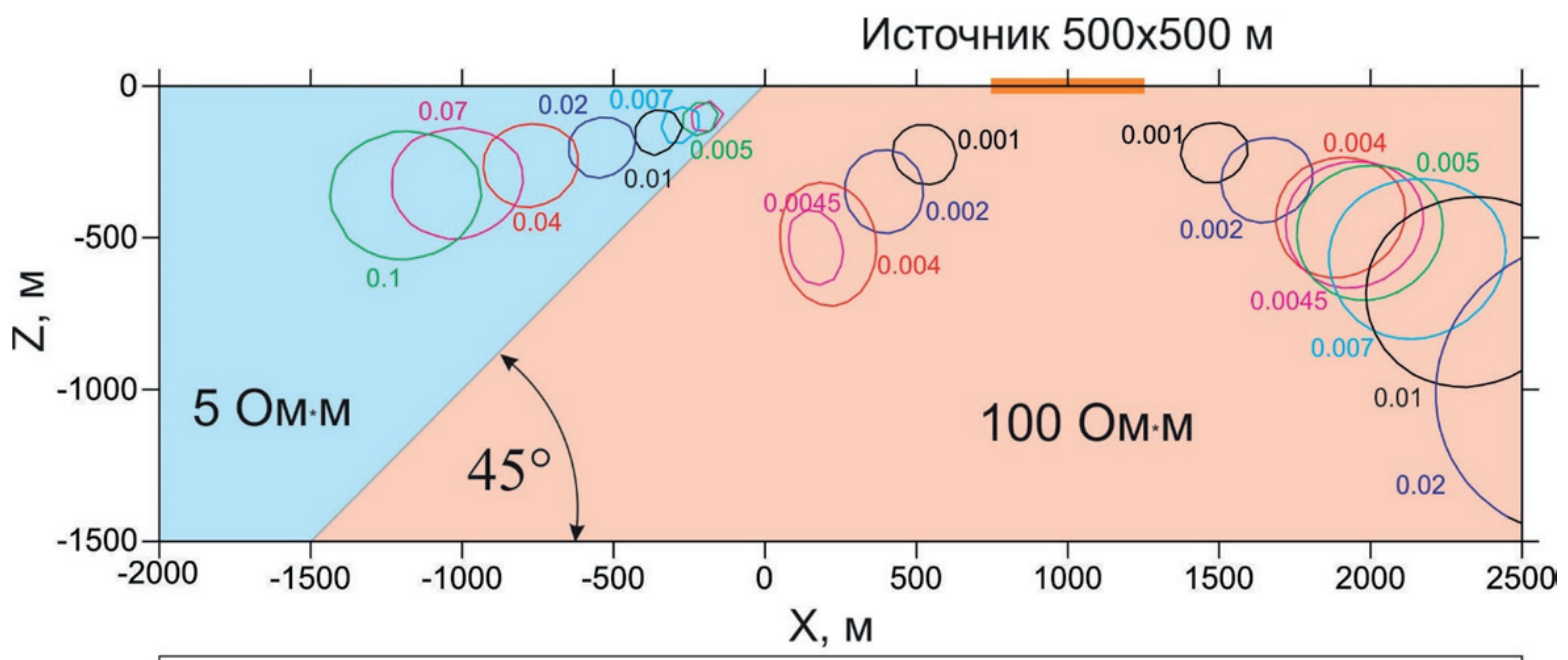

\subsection{2 - Изолиния 10\%-ой окрестности максимумальной амплитуды электрического поля в момент времени 0.02с.}

Рис. 2. Модель среды и изолинии 10\%-ой окрестности максимумальной алплитуды электрического поля. Источник над частью среды с УЭС 100 Ом'м

Fig. 2. Model of medium and contour lines of $10 \%$ locality for amplitude maximum of electric field. The field source is located above the part of the medium with $100 \mathrm{Ohm} \cdot \mathrm{m}$ electical resestivity 


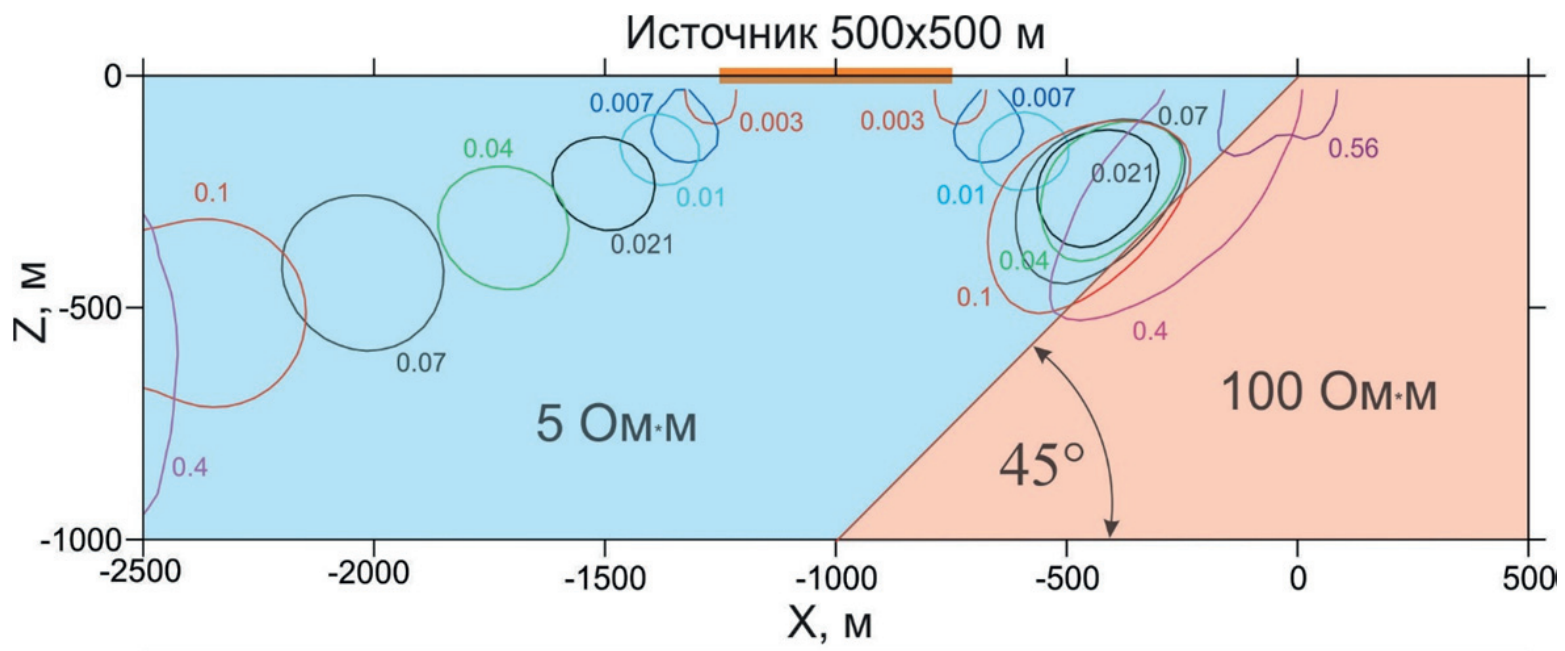

\section{- Изолиния $10 \%$-ой окрестности максимумальной амплитуды электрического поля в момент времени $0.01 \mathrm{c}$.}

Рис. 3. Модель среды и изолинии 10\%-й окрестности максимулальной амплитуды электрического поля. Источник над частью среды с УЭС 5 Ол:м

Fig. 3. Model of medium and contour lines of $10 \%$ locality for amplitude maximum of electric field. The field source is located above the part of the medium with $5 \mathrm{Ohm} \cdot \mathrm{m}$ electical resestivity

электрического вихря и его дальнейшем распространении так же, как в полупространстве с УЭС 5 Ом·м. Отметим при этом, что скорости погружения центров электрических вихрей уменьшаются: для левого центра с $4 \cdot 10^{5}$ до $1 \cdot 10^{5} \mathrm{M} / \mathrm{c}(100$ Ом·м) и с $5,2 \cdot 10^{4}$ до $0,35 \cdot 10^{4} \mathrm{M} / \mathrm{c}(5$ Ом·м); для правого центра с $4 \cdot 10^{5}$ до $0,53 \cdot 10^{5} \mathrm{~m} / \mathrm{c}$. Таким образом, если в первой фазе распространения центры электрических вихрей лежат в горизонтальной плоскости, то во второй фазе они лежат в плоскости, наклоненной в сторону менее проводящей среды.

На рис. 3 наблюдается существенно отличная картина. Видно, что до момента времени $t=4,5$ мс левая часть электрического вихря распространяется так же, как в однородном полупросранстве с УЭС 5 Омм (первая фаза распространения). Отметим при этом, что скорость погружения левого центра уменьщается с $5,2 \cdot 10^{4}$ до $0,35 \cdot 10^{4} \mathrm{M} / \mathrm{c}$. Тогда как на траекторию движения правого центра уже с ранних времён ( $t=7 \mathrm{mc}$ ) начинают влиять поверхностные заряды, индуцированные на наклонной границе. Во второй фазе распространения правый центр электрического вихря движется вдоль наклонного контакта, медленно диффундируя в слабопроводящий сектор нижнего полупространства.

Таким образом, использование для решения задач инверсии таких кинематических характеристик, как скорости распространения электрических вихрей, не представляется возможным из-за их сильной зависимости от времени.

На рис. 4 приведены временные развёртки ЭДС, наведённых в приёмниках текущими в среде вторичными вихревыми токами и поверхностными зарядами, при различных расстояниях (разно- сах) от источника до приёмника (шифр кривых). Разнос 3000 м соответствует выходу наклонного контакта на поверхность на линии $(\mathrm{X}=\mathrm{Z}=0 \mathrm{~m})$.

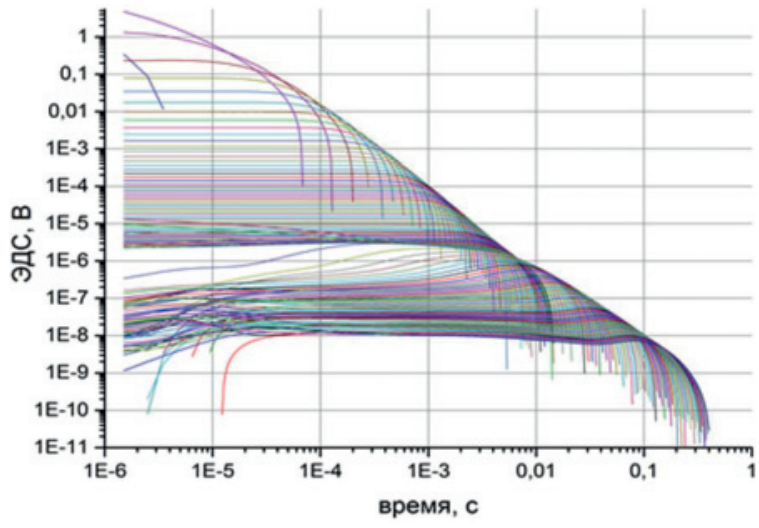

Pис. 4. ЭДС в многоразносной установке (наклон границы $30^{\circ}$ )

Fig. 4. EMF for multispacing array ( $30^{\circ}$ incline of boundary)

В соответствии с (1) для установки с фиксированным разносом были вычислены псевдоволновые трансформанты в зависимости от приведённого времени. Алгоритм расчётов описан в работах $[18,19]$. На рис. 5 показан их сводный набор для многоразносной установки.

Похожая картина наблюдается для границы с наклоном $45^{\circ}$ (рис. 6). При этом экстремумы псевдоволновых трансформант изменяют своё положение при переходе приёмников через наклонную границу (разнос 3000 м).

Построим годографы псевдоволновых трансформаций (рис. 7), связав $q$-время его экстремума (ось ординат) и расстояние между источником и 


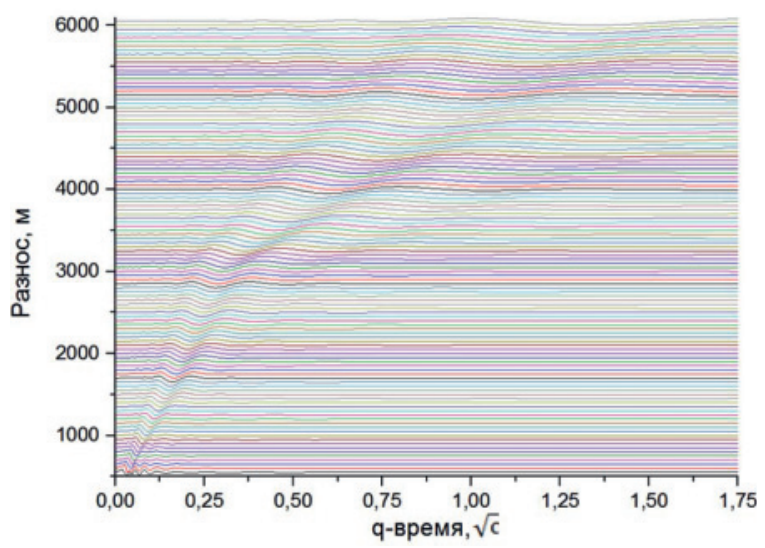

Рис. 5. Псевдоволновые трансфорланты (наклон границы $30^{\circ}$ )

Fig. 5. Pseudowave transformants ( $30^{\circ}$ incline of boundary)

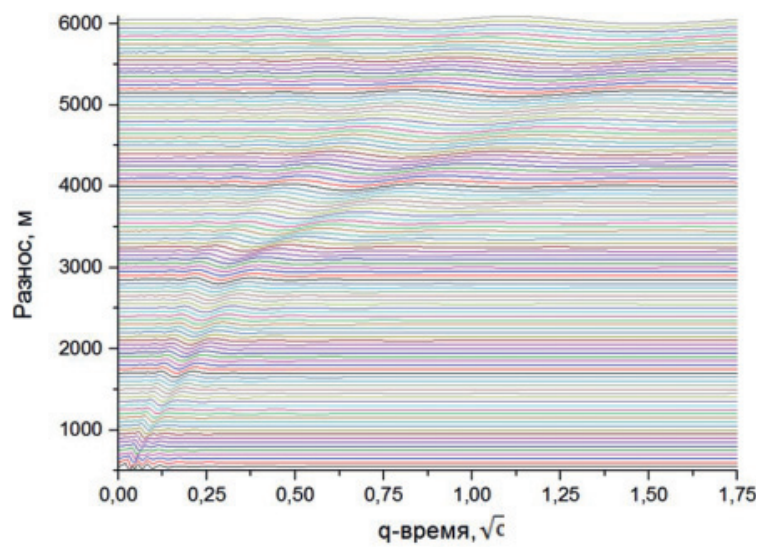

Pис. 6. Псевдоволновые трансформанты (наклон границы $45^{\circ}$ )

Fig. 6. Pseudowave transformants ( $45^{\circ}$ incline of boundary)

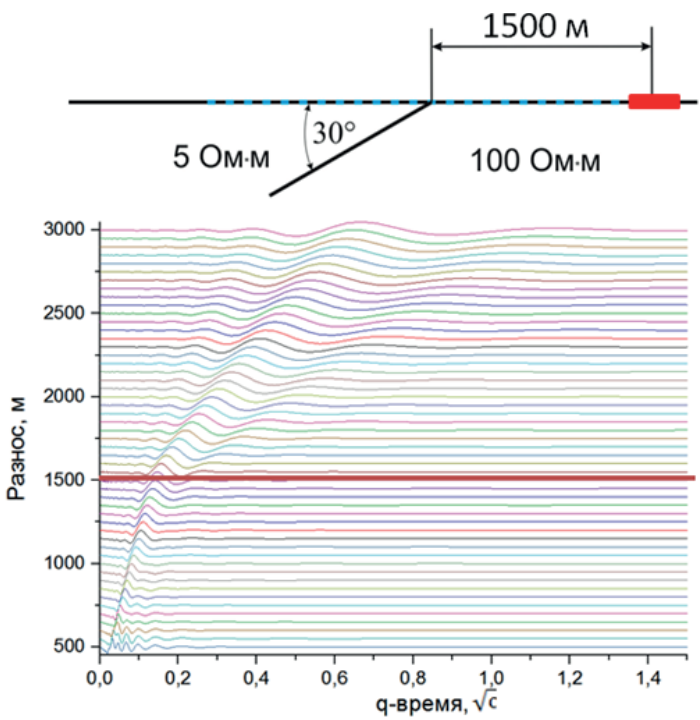

каждым приёмником (ось абсцисс). На годографах отчетливо выделяется выход контакта на поверхность - координата 3000 м. Левые части годографов (от 500 до 3000 м) для сред с различным углом наклона границы одинаковы и совпадают с годографом на поверхности проводящего полупространства с УЭС 100 Ом·м. После перехода приёмников через границу годографы для различных углов расходятся между собой. При этом угол наклона годографа возрастает с увеличением наклона границы.

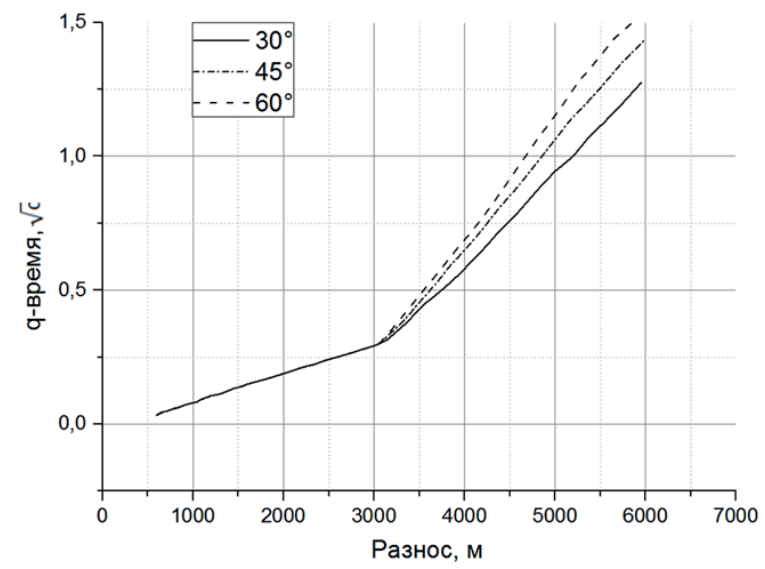

Puс. 7. Годографы в среде с наклонной границей (шифр кривыхугол наклона)

Fig. 7. Travel-time plot for the medium with incliened boundary (legend - incline on boundary)

На рис. 8 показаны две зеркальные расстановки источника и приёмников относительно линии выхода контакта на поверхность, а также соответствующие псевдоволновые трансформанты. Крас-
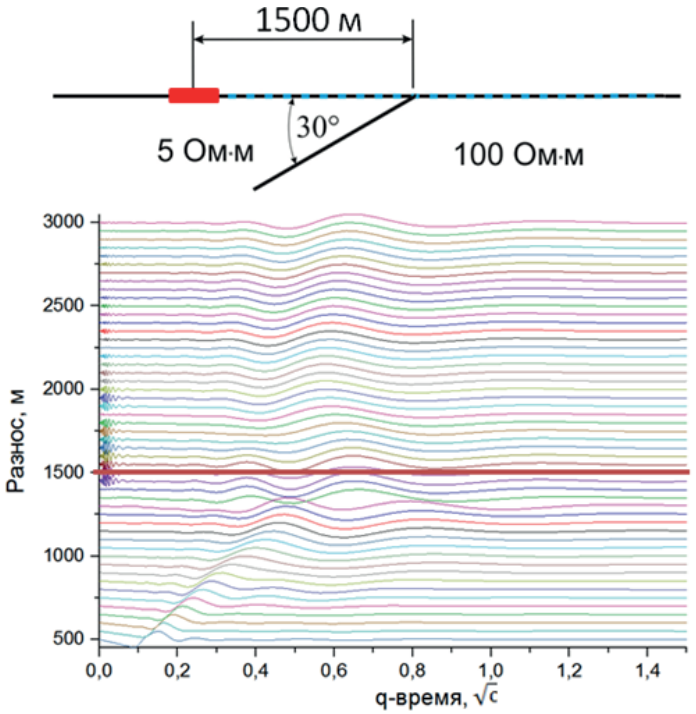

Рис. 8. Псевдоволновые трансфорланты (источник располагается в 1500 м (слева или справа) от места выхода наклонного контакта на поверхность)

Fig. 8. Pseudowave transformants (field source is located at $1500 \mathrm{~m}$ (left or right) from the exit to the surface of inclined boundary) 

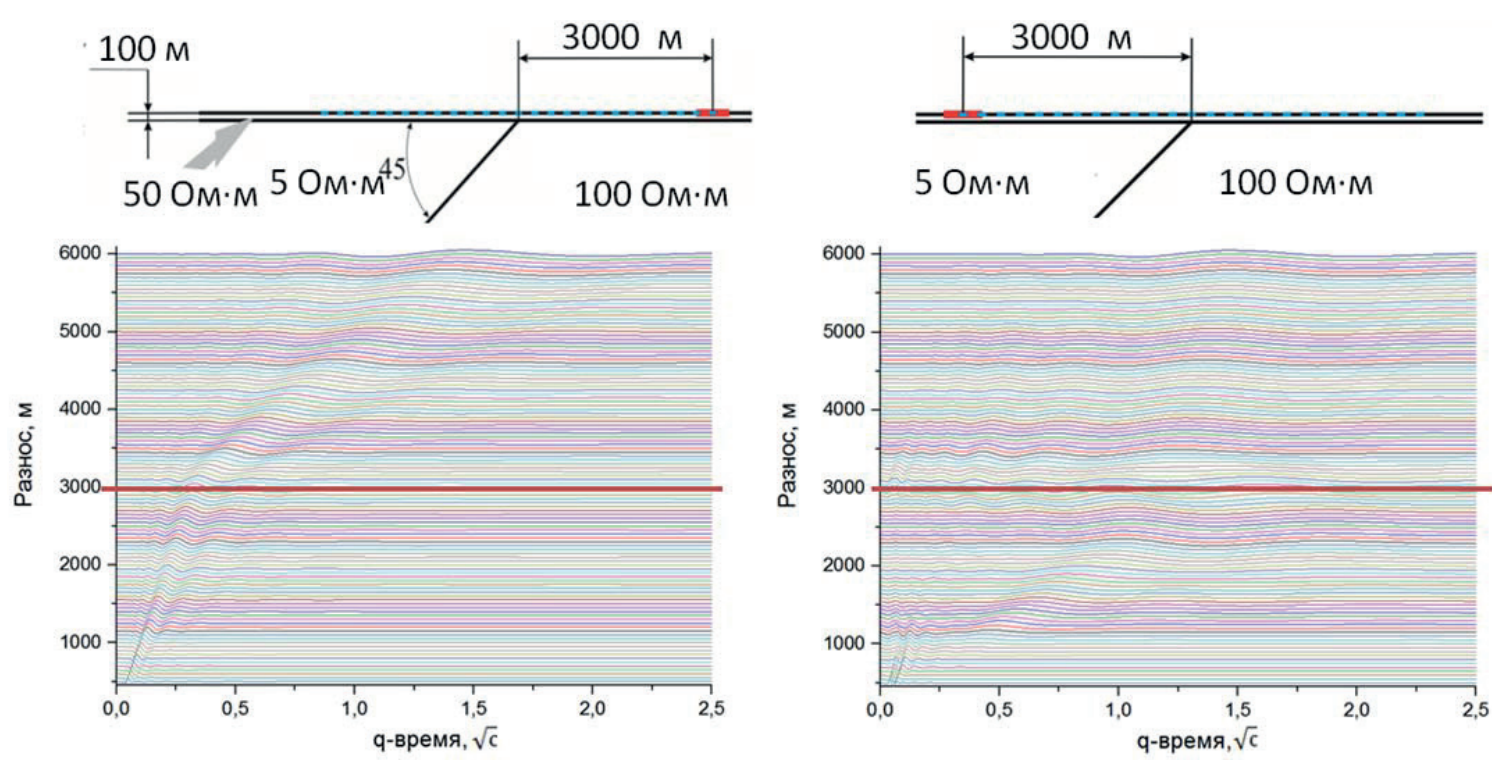

Рис.9. Псевдоволновые трансформанты (источник располагается в 3000 м (слева или справа) от места выхода наклонного контакта на поверхность)

Fig. 9. Pseudowave transformants (field source is located at $3000 \mathrm{~m}$ (left or right) from the exit to the surface of inclined boundary)

ными линиями выделено место выхода наклонного контакта на дневную поверхность.

Рассмотрим ту же самую модель с добавлением относительно маломощных (100 м) наносов с УЭС 50 Ом·м. На рис. 9 показаны псевдоволновые волнововые трансформанты.

Наносы практически не влияют на определение места выхода наклонного контакта, если источник расположен над высокоомным сектором среды. Если же источник находится на поверхности более проводящего сектора, то выход контакта под наносы становится практически незаметным.

\section{Заключение}

Вторичные вихревые токи, индуцированные в проводящей среде после отключения источника,

\section{СПИСОК ЛИТЕРАТУРЫ}

1. Кауфман А.А., Морозова Г.М. Теоретические основы метода зондирований становлением поля в ближней зоне. - Новосибирск: Наука, Сибирское отд., 1970. - 123 с.

2. Сидоров В.А. Импульсная электроразведка. - М.: Недра, 1985. - 192 c.

3. Эпов М.И., Ельцов И.Н. Прямые и обратные задачи индуктивной геоэлектрики в одномерных средах. - Препринт ОИГГиМ CO PAH. - 1992. - № 2. - 31 c.

4. Программное обеспечение Модем 3D для интерпретации данных нестационарных зондирований с учётом эффектов вызванной поляризации / М.И. Иванов, В.А. Катешов, И.А. Кремер, М.И. Эпов // Записки Горного института. - 2009. - Т. 183. C. $242-245$.

5. Использование свободных сетевых ресурсов предприятия для решения ёмких вычислительных геофизических задач / А.С. Мартьянов, Д.В. Тейтельбаум, К.С. Сердюк, А.А. Власов, И.Н. Ельцов // Каротажник. - 2011. - № 11 (209). - С. 56-64.

6. Physical and mathematical modeling of transient electromagnetic soundings over salt-dome structures / N.Yu. Bobrov, S.S. Kry- представляют собой замкнутые тороидальные структуры. Центры их сечений движутся в среде со скоростью, зависящей от времени, что не позволяет их использовать при инверсии в качестве кинематических характеристик.

Введение псевдоволновых трансформаций позволяет получать годографы из данных метода ЗС, зарегистрированных на линейных профилях. Наборы псевдоволновых трансформаций, построенные в координатах «разнос - приведённое время», позволяют даже на качественном уровне выделить линию выхода наклонного контакта на дневную поверхность. Перекрывающие наносы ослабляют максимумы псевдоволновых трансформаций и снижают возможность точной локализации линии выхода наклонной границы.

lov, E.Yu. Antonov, A.N. Shein, N.P. Smilevets // Russian Geology and Geophysics. - 2017. - V. 58. - P. 266-274.

7. Lee S., Memechan G.A. Phase-field imaging: the electromagnetic equivalent of seismic migration // Geophysics. - 1987. № 52 (5). - P. 678-693.

8. Lee K.H. A new approach to modeling the electromagnetic response of conductive medium // Geophysics. - 1989. - № 54 (9). P. 1180-1192.

9. Lee K.H., Xie G. A new approach to imaging with low-frequency electromagnetic fields // Geophysics. - 1993. - № 58. P. 780-796.

10. Levy S., Oldenburg D., Wang J. Subsurface imaging using magnetotelluric data // Geophysics. - 1988. - № 53. - P. 104-117.

11. Gilbert D., Virieux J. Electromagnetic imaging and simulated annealing // Geophysics. - 1991. - № 96. - P. 8057-8067.

12. Gershenson M. Simple interpretation of time-domain electromagnetic sounding using similarities between wave and diffusion propagation // Geophysics. - 1997. - № 62 (3). - P. 763-774.

13. Swidinsky A. Transient Electromagnetic Modelling and Imaging of Thin Resistive Structures: Applications for Gas Hydrate As- 
sessment // A thesis submitted in conformity with the requirements for the degree of Doctor of Philosophy Department of Physics University. -Toronto, 2011. $-255 \mathrm{p}$.

14. An optimized method for transient electromagnetic field-wave field conversion / X. Li, G.Q. Xue, J.P. Song, W.B. Guo, J.J. Wu // Chin J. Geophys. - 2005. - № 48 (5). - P. 1185-1190.

15. Xue G.Q., Yan Y.J., Li X. Control of the waveform dispersion effect and applications in a TEM imaging technique for identifying underground objects // J. Geophys. Eng. 8. - 2011. P. 195-201.

16. Li X., Xue G., Yin C. Migration imaging of the transient electromagnetic method. - Beijing: Science Press, 2016. - 139 p.

17. Xue G.Q., Bai C.Y., Li X. Extracting virtual reflection wave from TEM data based on regularizing method // Pure appl. Geophys. 2012. - № 69 (7). - P. 1269-1282.
18. Грецков Г.А., Эпов М.И., Антонов Е.Ю. Алгоритмы и программы для преобразования данных зондирования становлением поля в пространство решений волнового уравнения // Вопросы естествознания. - 2016. - № 1 (9). - С. 115-119.

19. Gretskov G.A., Epov M.I., Antonov E.Yu. Wave transforms of transient electromagnetic field in conductive earth // Russian Geology and Geophysics. - 2017. - V. 58 (201). - P. 744-751.

20. Approximation of a near-vertical boundary in the problems of pulsed electromagnetic soundings / N.V. Shtabel', M.I. Epov, E.Yu. Antonov, M.A. Korsakov // Russian Geology and Geophysics. -2014 . - V. 55. - P. 89-97.

Поступила 23.11.2018 2.

\section{Информация об авторах}

Греиков $\Gamma . A$., аспирант, инженер Института нефтегазовой геологии и геофизики им. А.А. Трофимука Сибирского отделения РАН.

Эпов М.И., доктор технических наук, профессор, академик РАН, главный научный сотрудник Института нефтегазовой геологии и геофизики им. А.А. Трофимука Сибирского отделения РАН.

IIеин A.H., кандидат физико-математических наук, старший научный сотрудник, Института нефтегазовой геологии и геофизики им. А.А. Трофимука Сибирского отделения РАН. 
УДК 550.837 .6

\title{
APPLYING PSEUDOWAVE TRANSFORMATION IN TRANSIENT ELECTROMAGNETIC PROSPECTING FOR RECOGNITION OF INCLINED GEOLOGICAL BOUNDARIES
}

\author{
Gleb A. Gretskov', \\ Greckov.Gleb@gmail.com \\ Mikhail I. Epov', \\ EpovMl@ipgg.sbras.ru \\ Aleksandr N. Shein', \\ SheinAN@ipgg.sbras.ru \\ 1 Institute of Petroleum Geology and Geophysics named after A.A. Trofimuk SB RAS,
3, Academician Koptueg Avenue, Novosibirsk, 630090, Russia.
}

Relevance. Transient electromagnetic soundings is the method in-demand of electric survey applied for mineral deposits prospecting. Modern geoelectric problems require the usage of multichannel devices. For such devices the processing and interpretation problem becomes very resource intensive. Thus, the further development of interpretation methods is highly recommended. One of the possible ways of such development is the usage of wavefield transformation for transient electromagnetic data.

The main aim of the research is to apply the wavefield transformation to the transient electromagnetic data measured in multireceivers systems in conductive media with inclined boundary for obtaining new qualitative estimations for the geological media on study. Method of research is based on new numerical algorithms for the stable wavefiled transformations of transient electromagnetic soundings data. Input data for transformation checkout (calculated three-dimensional curves of transient electromagnetic field response) was received using MODEM3D software package, which is designed for direct modeling of transient electromagnetic fields.

Results. Pseudowave transformations of transient electromagnetic soundings for the conductive media with inclined boundary were obtained. These pseudowave transformations are used to plot travel-time curves. The resulting sets of transformations allow allocating the outcrop of the inclined contact on the surface. It is established that overlapping sediments weaken the maxima of pseudowave transformations and reduce the possibility of exact localization of the outcrop of the inclined contact. The behavior of toroidal vertical currents depending on time in the media with inclined boundary was considered.

\section{Key words:}

Transient electromagnetic soundings, pseudowave transform, Q-transform, three-dimensional problem, medium with inclined boundary, hodograph.

\section{REFERENCES}

1. Kaufman A.A., Morozova G.M. Teoreticheskie osnovy metoda zondirovaniy stanovleniem polya $v$ blizhney zone [Theoretical bases of the near-field transient electromagnetic sounding method]. Novosibirsk, Nauka Publ., 1970.123 p.

2. Sidorov V.A. Impulsnaya elektrorazvedka [Pulse electric sounding]. Moscow, Nedra Publ., 1985. 192 p.

3. Epov M.I., Eltsov I.N. Pryamye i obratnye zadachi induktivnoy geoelektriki v odnomernykh sredakh [Direct and inverse problems of inductive geoelectrics in one-dimensional media]. Preprint OIGGiM SO RAN, 1992, no. 2, $31 \mathrm{p}$.

4. Ivanov M.I., Kateshov V.A., Kremer I.A., Epov M.I Modem 3D new software for the interpretation of IP-affected 3D tem data. Journal of Mining Institute, 2009, vol. 183, pp. 242-245. In Rus.

5. Martyanov A.S., Teytelbaum D.V., Serdjuk K.S., Vlasov A.A., Eltsov I.N. Using vacant network resources of a company for solving geophysical problems requiring much computation. Sci-tech bulletin Karotazhnik (Well Logger), 2011, no. 11 (209), pp. 56-64. In Rus.

6. Bobrov N.Ju., Krylov S.S., Antonov E.Yu., Shein A.N., Smilevec N.P. Physical and mathematical modeling of transient electromagnetic soundings over salt-dome structures. Russian Geology and Geophysics, 2017, vol. 58, pp. 266-274.

7. Lee S., Memechan G.A. Phase-field imaging: the electromagnetic equivalent of seismic migration. Geophysics, 1987, no. 52 (5), pp. 678-693.

8. Lee K.H. A new approach to modeling the electromagnetic response of conductive medium. Geophysics, 1989, № 54 (9), pp. 1180-1192.
9. Lee K.H., Xie G. A new approach to imaging with low-frequency electromagnetic fields. Geophysics, 1993, no. 58, pp. 780-796.

10. Levy S., Oldenburg D., Wang J. Subsurface imaging using magnetotelluric data. Geophysics, 1988, no. 53, pp. 104-117.

11. Gilbert D., Virieux J. Electromagnetic imaging and simulated annealing. Geophysics, 1991, no. 96, pp. 8057-8067.

12. Gershenson M. Simple interpretation of time-domain electromagnetic sounding using similarities between wave and diffusion propagation. Geophysics, 1997, no. 62 (3), pp. 763-774.

13. Swidinsky A. Transient Electromagnetic Modelling and Imaging of Thin Resistive Structures: Applications for Gas Hydrate Assessment. A thesis submitted in conformity with the requirements for the degree of Doctor of Philosophy Department of Physics University. Toronto, 2011. $255 \mathrm{p}$.

14. Li X., Xue G.Q., Song J.P., Guo W.B., Wu J.J. An optimized method for transient electromagnetic field-wave field conversion. Chin J. Geophys., 2005, no. 48 (5), pp. 1185-1190.

15. Xue G.Q., Yan Y.J., Li X. Control of the waveform dispersion effect and applications in a TEM imaging technique for identifying underground objects. J. Geophys. Eng. 8, 2011, pp. 195-201.

16. Li X., Xue G., Yin C. Migration imaging of the transient electromagnetic method. Beijing, Science Press, 2016. 139 p.

17. Xue G.Q., Bai C.Y., Li X. Extracting virtual reflection wave from TEM data based on regularizing method. Pure appl. Geophys., 2012, no. 69 (7), pp. 1269-1282.

18. Gretskov G.A., Epov M.I., Antonov E.Yu. Algorithms and programs for data conversion sounding in the space of solutions of the wave equation. Voprosy estestvoznaniya, 2016, no. 1 (9), pp. 115-119. In Rus. 
19. Gretskov G.A., Epov M.I., Antonov E.Yu. Wave transforms of transient electromagnetic field in conductive earth. Russian Geology and Geophysics, 2017, vol. 58 (201), pp. 744-751.

20. Shtabel N.V., Epov M.I., Antonov E.Yu., Korsakov M.A. Approximation of a near-vertical boundary in the problems of pulsed electromagnetic soundings. Russian Geology and Geophysics, 2014, vol. 55, pp. 89-97.

Received: 23 November 2018.

\section{Information about the authors}

Gleb A. Gretskov, postgraduate, engineer, Institute of Petroleum Geology and Geophysics named after A.A. Trofimuk SB RAS.

Mikhail I. Epov, Dr. Sc., professor, RAS academician, senior researcher, Institute of Petroleum Geology and Geophysics named after A.A. Trofimuk SB RAS.

Aleksandr N. Shein, Cand. Sc., senior researcher, Institute of Petroleum Geology and Geophysics named after A.A. Trofimuk SB RAS. 